\title{
Structure-antioxidant Activity (Oxygen Radical Absorbance Capacity) Relationships of Phenolic Compounds
}

\section{Shuhei Sakurai}

Yokohama National University: Yokohama Kokuritsu Daigaku

Yuta Kawakami

Yokohama National University: Yokohama Kokuritsu Daigaku

Manabu Kuroki

Yokohama National University: Yokohama Kokuritsu Daigaku

Hiroaki Gotoh ( $\nabla$ gotoh-hiroaki-yw@ynu.ac.jp)

Yokohama National University https://orcid.org/0000-0002-1920-6020

\section{Research Article}

Keywords: oxygen radical absorbance capacity, bond dissociation enthalpy, clusterwise linear regression analysis, structure-activity relationship, antioxidant capacity, phenolic compounds

Posted Date: February 15th, 2021

DOl: https://doi.org/10.21203/rs.3.rs-180961/v1

License: (9) This work is licensed under a Creative Commons Attribution 4.0 International License.

Read Full License 
Structure-antioxidant activity (oxygen radical absorbance capacity) relationships of phenolic compounds

\author{
S. Sakurai, Y. Kawakami, M. Kuroki, H. Gotoh* \\ Department of Chemistry and Life Science, Yokohama National University, 79-5 Tokiwadai, Hodogaya- \\ ku, Yokohama 240-8501, Japan \\ Tel.: +81-(0)45-339-3964 \\ Fax: +81-(0)45-339-3964 \\ ORCID iD: 0000-0002-1920-6020 \\ Email: gotoh-hiroaki-yw@ynu.ac.jp
}




\begin{abstract}
Antioxidant capacity is the extent to which a compound can eliminate reactive oxygen species, and in vitro methods for its chemical evaluation have been proposed. Among these methods, the oxygen radical absorbance capacity (ORAC) assay comes close to the oxidation reaction in the living body because it generates radical species that mimic the lipid peroxyl radical involved in the peroxidation reaction of biological components and react in a phosphate buffer. In this study, PM7, a semi-empirical molecular orbital method, was used to calculate the thermodynamic properties (bond dissociation enthalpy, ionisation potential, and proton affinity) associated with ORAC. We also applied the clusterwise linear regression analysis as a statistical method for grouping the antioxidants by structure. By analysing the data for antioxidants, the trend in the hydrophilic ORAC values was determined using the calculated structures and bond dissociation enthalpies of the groups classified according to the presence or absence of oxygen functional groups in the ortho position of phenol. Further studies of indicators other than bond dissociation enthalpy are needed to predict the ORAC of other antioxidants such as flavonoids and indoles.
\end{abstract}

Keywords: oxygen radical absorbance capacity; bond dissociation enthalpy; clusterwise linear regression analysis; structure-activity relationship; antioxidant capacity; phenolic compounds

\title{
1. Introduction
}

Part of the oxygen taken up by respiration is externally stimulated and becomes reactive oxygen species (ROS) [1]. Phenolic compounds are generally known to remove ROS via the hydrogen atom transfer (HAT, Eq. 1) [2], electron transfer-proton transfer (ET-PT, Eq. 2) [3], or sequential proton loss-electron transfer (SPLET, Eq. 3) mechanism [4].

$$
\begin{aligned}
& \mathrm{ArOH}+\mathrm{ROO}^{\circ} \rightarrow \mathrm{ArO}^{\circ}+\mathrm{ROO}-\mathrm{H} \\
& \mathrm{ArOH}+\mathrm{ROO}^{\circ} \rightarrow \mathrm{ArOH}^{+}+\mathrm{ROO}^{-} \\
& \mathrm{ArOH}^{+}+\mathrm{ROO}^{-} \rightarrow \mathrm{ArO}^{\cdot}+\mathrm{ROO}-\mathrm{H} \\
& \mathrm{ArOH} \rightarrow \mathrm{ArO}^{-}+\mathrm{H}^{+} \\
& \mathrm{ArO}^{-}+\mathrm{ROO}^{\cdot} \rightarrow \mathrm{ArO}^{\cdot}+\mathrm{ROO}^{-}
\end{aligned}
$$

Antioxidant capacity is the extent to which a compound can eliminate ROS, and in vitro methods for its chemical evaluation have been proposed [5]. One of these methods, the oxygen radical absorbance capacity (ORAC) assay [6], evaluates the suppression of fluorescence due to the decomposition of the fluorescent probe by the peroxy radical derived from 2,2'-azobis(2- 
amidinopropane)dihydrochloride. The ORAC assay comes close to the oxidation reaction in the living body because it generates radical species that mimic the lipid peroxyl radical involved in the peroxidation reaction of biological components and react in a phosphate buffer [7]. Furthermore, this method can be applied in the medical field because it can measure the antioxidative ability of biological samples such as serum [8]. In addition, water-soluble [9,10] and lipophilic [10] antioxidants can be measured by the hydrophilic ORAC (H-ORAC) and lipophilic ORAC assays, respectively, using a solution added to the phosphate buffer. The ORAC assay mainly evaluates radical scavenging ability, which proceeds through the HAT mechanism [11]. However, quantum chemical analysis of flavonoid structures and the corresponding ORAC values suggested that the SPLET mechanism, which is related to the proton affinity (PA) and electron transfer enthalpy acquired for the electron transfer sequence, is also involved [12].

Villaño et al. [13] tested the antioxidant components of wine samples using the ABTS (2,2'-azinobis(3-ethylbenzthiazoline-6-sulfonic acid)), DPPH (2,2-diphenyl-1-picryl-hydrazyl-hydrate), and ORAC assays. They reported that catechol and guaiacol sites have high ORAC values, while pyrogallol and 2,6-dimethoxyphenol sites have low ORAC values. In addition, 2-phenylpropionic acid, which does not have a phenolic hydroxyl group, was discovered to exhibit antioxidant capacity. However, they did not explain the trend in ORAC values and the cause of the antioxidant capacity of compounds without a phenolic hydroxyl group. Rodriguez-Naranjo et al. [14] tested melatonin and its derivatives using the ferric reducing/antioxidant power (FRAP), DPPH, and ORAC assays. The FRAP and DPPH values were reported to increase in the presence of 5-OH in the indole ring, but the ORAC value did not show such a tendency [15]. However, it was not discussed in detail how a few hydroxylfree derivatives had high ORAC values because of the reaction of the - $\mathrm{NH}$ group in the indole ring with peroxyl radicals [16].

In recent years, efforts have been made to obtain a structure-activity relationship using structural formula and antioxidant capacity obtained by quantum chemical calculations. For example, Ordoudi et al. [17] investigated the relationship of the $\mathrm{DPPH} \bullet$ scavenging activity with the bond dissociation enthalpy (BDE) and Hammett and Brown parameters. In addition, the DPPH value, BDE, and ionisation potential (IP) have been shown to have a structure-activity relationship [18, 19]. On the other hand, there are only a few studies on the structure-activity relationship of ORAC with calculated thermodynamic properties. Slavova-Kazakova et al. [20] showed that there is a negative correlation between the ORAC and BDE values obtained by theoretical calculations at the UB3LYP/6-31+G(d,p) level. Žuvela et al. [21] developed quantitative structure-activity relationship models to predict the ORAC values of flavonoids. To our knowledge, the structure-activity relationship with the ORAC values of general antioxidants has not been obtained to date.

In this study, we analysed the structure-activity relationship between the ORAC values and thermodynamic properties obtained by theoretical calculations for compounds containing phenols, as 
well as melatonin and its derivatives, which were grouped by structural characteristics. We also used clusterwise linear regression analysis, which was proposed by Späth [22-24] and extended by Vicari and Vichi [25], to analyse the data. It is a multivariate statistical method that simultaneously builds regression models for each cluster and classifies compounds into several clusters to improve the explanatory power for a response variable and construct homogenous groups. We described in detail the BDE, which is the barrier of the HAT mechanism that is believed to drive the process in the ORAC assay.

2. Materials and methods

We analysed the 30 antioxidants, excluding rutin, investigated by Villaño et al. [13] and 12 antioxidants, including melatonin and its derivatives, investigated by Rodriguez-Naranjo et al. [14]. Differences between the H-ORAC values obtained from the two experiments were considered to be minor because the experiments were performed in the same laboratory. The 42 compounds were classified into the following 7 groups on the basis of their structural characteristics. Representative examples of compounds from each group are shown in Table 1.

Group 0: phenols di-substituted at the ortho position

Group 1: phenols mono-substituted at the ortho position

Group 2: phenols unsubstituted at the ortho position

Group 3: flavans

Group 4: flavonols

Group 5: phenylacetic acids

Group 6: hydroxyindoles

Group 7: indoles

\subsection{ORAC}

The García-Parrilla group measured the H-ORAC values of wine phenolic compounds and metabolites [13] and melatonin and related indoles [14] using B-phycoerythrin and fluorescein, respectively, as the fluorescent probe. Currently, the ORAC assay uses fluorescein, which gives highly reproducible results and is inexpensive. However, there is no large difference between the H-ORAC values due to the different fluorescent probes used $[28,29]$. In this study, we analysed the H-ORAC values reported in these two papers.

\subsection{Theoretical calculations}

For each compound, a conformational search was performed using the molecular force field calculation method of Balloon [26], and the most stable structure was taken as the initial structure. 
The semi-empirical calculations implemented in MOPAC [27] were performed using these initial structures. The PM7 method [28] was used for structural optimisation in vacuum. Amić and Lučić [29] reported that the PM6 method [30] has the same accuracy and speed as the density functional theory method [31]. The PM7 method is a further improvement of PM6 and was therefore used in this study. Using the enthalpy $(H)$ obtained from this calculation, three thermodynamic parameters, namely, BDE, IP, and PA, were calculated using Eqs. 4-6. The enthalpy in vacuum, $H\left(\mathrm{e}^{-}\right)$, is $0.7516 \mathrm{kcal} / \mathrm{mol}$ [32]. BDE, IP, and PA were chosen to support the HAT, ET-PT, and SPLET mechanisms, respectively. For BDE and PA, it was assumed that - $\mathrm{OH}(-\mathrm{CH})$, which has the smallest value, is more likely to react with the peroxyl radical.

$$
\begin{aligned}
& \mathrm{BDE}=\mathrm{H}(\mathrm{ArO})+\mathrm{H}\left(\mathrm{H}^{-}\right)-\mathrm{H}(\mathrm{ArOH}) \\
& \mathrm{IP}=\mathrm{H}\left(\mathrm{ArOH}^{+}\right)+\mathrm{H}\left(\mathrm{e}^{-}\right)-\mathrm{H}(\mathrm{ArOH}) \\
& \mathrm{PA}=\mathrm{H}\left(\mathrm{ArO}^{-}\right)+\mathrm{H}^{+}\left(\mathrm{H}^{+}\right)-\mathrm{H}(\mathrm{ArOH})
\end{aligned}
$$

\subsection{Clusterwise linear regression analysis}

Clusterwise linear regression analysis is a multivariate statistical method that simultaneously builds linear regression models for each cluster and classifies a whole set of compounds into several clusters to improve the explanatory power for a response variable and construct homogenous groups. This method is performed in four steps as described below (see Supporting Information for details).

Step 1: Given a whole set of compounds, classify it into mutually exclusive initial clusters $C_{1}, C_{2}, \ldots, C_{k}$.

Step 2: For the given mutually exclusive clusters $C_{1}, C_{2}, \ldots, C_{k}$, define the classification error and derive the cluster centre functions that minimize the classification error.

Step 3: Referring to the cluster centre functions in Step 2, re-assign each compound to one of the clusters to minimize the classification error.

Step 4: If none of the cluster centre functions have changed, finish the algorithm. Otherwise, replace $\tilde{C}_{l}$ with $C_{l}$ and return to step 2.

In this study, the BDE and H-ORAC values were taken as the explanatory variable $(q=1)$ and response variable, respectively. In addition, the sample size was $n=12$ because we were concerned with the characterisation of the clusters $(k=2)$, neighboring $1\left(n \_1=5\right)$, and neighboring $2\left(n \_2=7\right)$. Furthermore, noting that all compounds were labelled as neighboring, we used them as the labels of the initial clusters to reflect the characteristics of neighboring 1 and neighboring 2 on the data analysis.

\section{Results}

3.1. Relationship between thermodynamic parameters and H-ORAC 
Table 1 shows a typical example from each compound group. All the compounds are shown in Supporting Information Table S1. Figure 1 shows the correlation between the H-ORAC and minimum $\mathrm{BDE}$ value, which is the lowest among the $\mathrm{BDE}$ values of $\mathrm{C}-\mathrm{H}$ and $\mathrm{O}-\mathrm{H}$ bonds in a molecule. For groups 0,1 , and 2, the H-ORAC and minimum BDE values are correlated, that is, the H-ORAC value increases as the BDE decreases. IP and PA are not as relevant to H-ORAC as BDE (see supporting information Figure S1, S2). The relationship between BDE and H-ORAC is described in detail below.

For group 0 (phenols di-substituted at the ortho position), the H-ORAC value tends to decrease within the BDE range of 74-77 kcal/mol, although this is based on three samples. When the -OH of the adjacent substituent is changed to -OMe (comparison between gallic acid and syringic acid), the BDE decreases while the H-ORAC value increases (Fig. 1). In addition, when an unsaturated carbon chain is bonded to the para position (comparison between syringic acid and sinapinic acid), delocalisation of unpaired electrons decreases the BDE and increases the H-ORAC value (Fig. 1). This is consistent with the fact that $\alpha, \beta$-unsaturated carbon chains enhance the antioxidant capacity of the curcumin skeleton \{Formatting Citation\}. For group 1 (phenols mono-substituted at the ortho position), the H-ORAC value tends to decrease within the BDE range of 75-80 kcal/mol (Fig. 1). However, protocatechuic acid has a BDE similar to that of trans-ferulic acid and H-ORAC value similar to that of caffeic acid. For group 2 (phenols unsubstituted at the ortho position), the H-ORAC value tends to decrease within the BDE range of $80-86 \mathrm{kcal} / \mathrm{mol}$. The high $\mathrm{H}-\mathrm{ORAC}$ value of caffeic acid is due to the catechol site and $\alpha, \beta$-unsaturated carbon chain at the para position (Fig. 1).

If sites other than the phenolic $\mathrm{OH}$, for example, the benzyl site, has a low $\mathrm{BDE}$, it is difficult to find a correlation using only the minimum BDE calculated by PM7 (Fig. 1). Kondo et al. [34] showed that the BDE of the catechin dimer C-2 is lower than that of the phenolic hydroxyl group. In this study, this tendency is confirmed for the compound group having a flavan skeleton. The BDE of C-2 can be either 71 or $74 \mathrm{kcal} / \mathrm{mol}$ depending on the configuration of $\mathrm{OH}$ and arrangement of substituents in the compound. As can be seen from Fig. 1, the H-ORAC values of groups 3 (flavans) and 4 (flavonols) rise while the BDE is constant; thus, the H-ORAC value is independent of the minimum BDE. For both of these groups, it would be reasonable to consider that the H-ORAC value is determined by other compounds but not by the minimum BDE.

For group 5 (phenylacetic acids), (S)-(+)-2-phenylpropionic acid does not have ArOH, although the $\mathrm{BDE}$ of the benzylic $\mathrm{CH}$ is approximately the same as that of $\mathrm{ArOH}$ [13]. Because this site has a nonzero H-ORAC value $(0.41 \mathrm{~mol} \mathrm{TE} / \mathrm{g})$, it can be a reaction point. This is confirmed by the fact that the H-ORAC of 3-hydroxybenzoic acid is almost zero ( $0.01 \mathrm{~mol} \mathrm{TE} / \mathrm{g})$, whereas that of 3hydroxyphenylacetic acid is not $(0.42 \mathrm{~mol} \mathrm{TE} / \mathrm{g})$. On the other hand, for the compounds with catechol and guaiacol sites, the increase in H-ORAC value is more significant than the decrease in BDE. As a result, the H-ORAC value of 3,4-dihydroxyphenylacetic acid increases. 
Analysis of groups 6 (indoles) and 7 (hydroxyindoles) shows that the change in the H-ORAC value is not as significant that in the BDE, except in the cases of Mel and 6-Mel. Because the calculated $\mathrm{BDE}$ of the $-\mathrm{COOH}$ group of the side chain of Trp and 5_MeOH_IAA is low, plotting the minimum $\mathrm{BDE}$ gives a constant $\mathrm{H}-\mathrm{ORAC}$ value. However, since the $\mathrm{BDE}$ of the $-\mathrm{NH}$ group in the indole ring is approximately $88 \mathrm{kcal} / \mathrm{mol}$, it can be inferred that the - $\mathrm{COOH}$ group is not involved in the reaction. Moreover, on the basis of the minimum BDE, the high H-ORAC values of Mel and 6-Mel cannot be explained. 
Table 1 Representative examples of compounds used to analyse the relationship between the experimental hydrophilic oxygen radical absorbance capacity (H-ORAC) and thermodynamic parameters obtained by theoretical calculations ${ }^{a}$

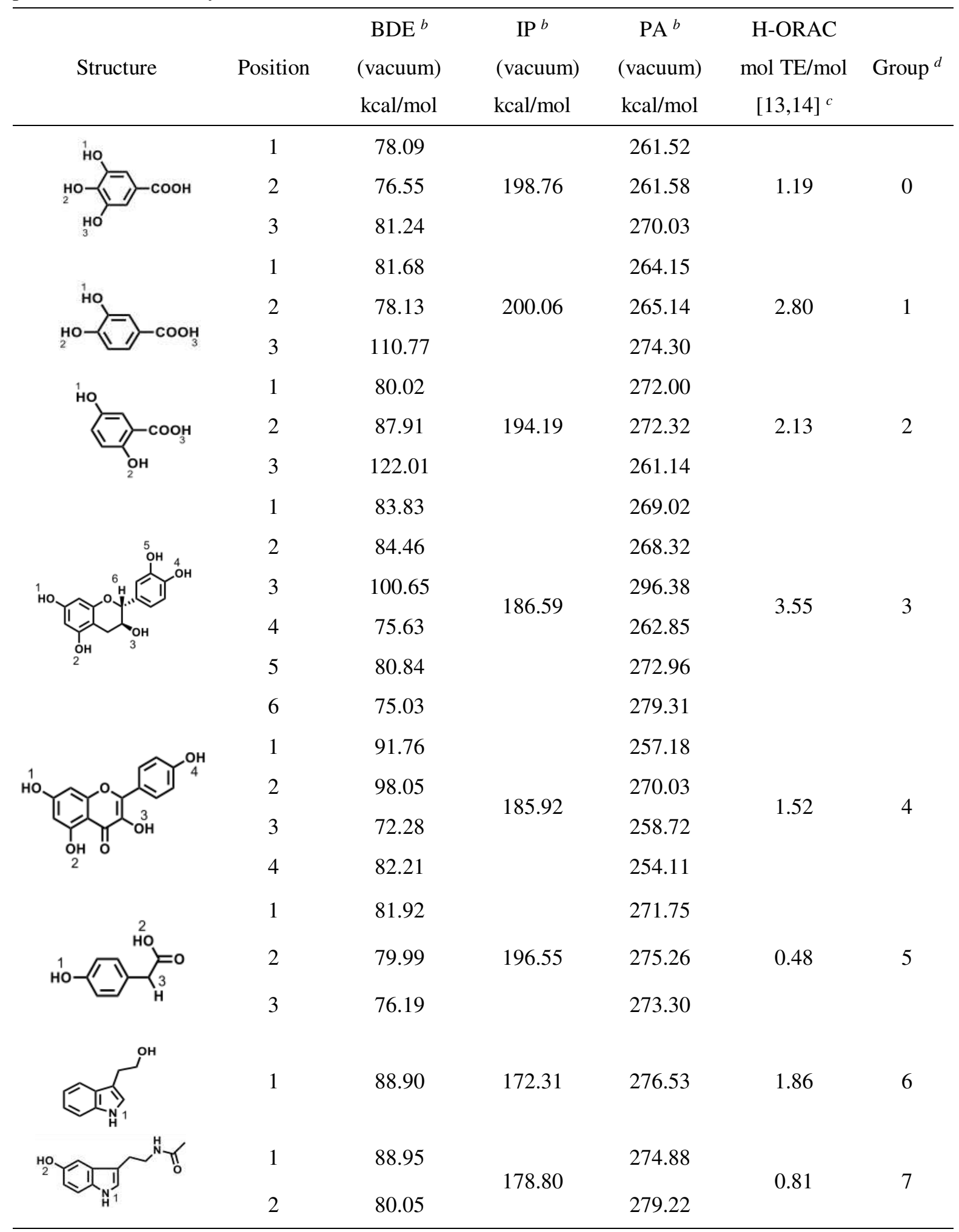


${ }^{a}$ All compounds are shown in Table S1 of the Supporting Information. ${ }^{b}$ BDE: bond dissociation enthalpy, IP: ionisation potential, PA: proton affinity. ${ }^{c}$ The García-Parrilla group measured the HORAC values of wine phenolic compounds and metabolites [13] and melatonin and related indoles [14]. ${ }^{d}$ Group 0: phenols di-substituted at the ortho position, Group 1: phenols mono-substituted at the ortho position, group 2: phenols unsubstituted at the ortho position, group 3: flavans, group 4: flavonols, group 5: phenylacetic acids, group 6: hydroxyindoles, group 7: indoles

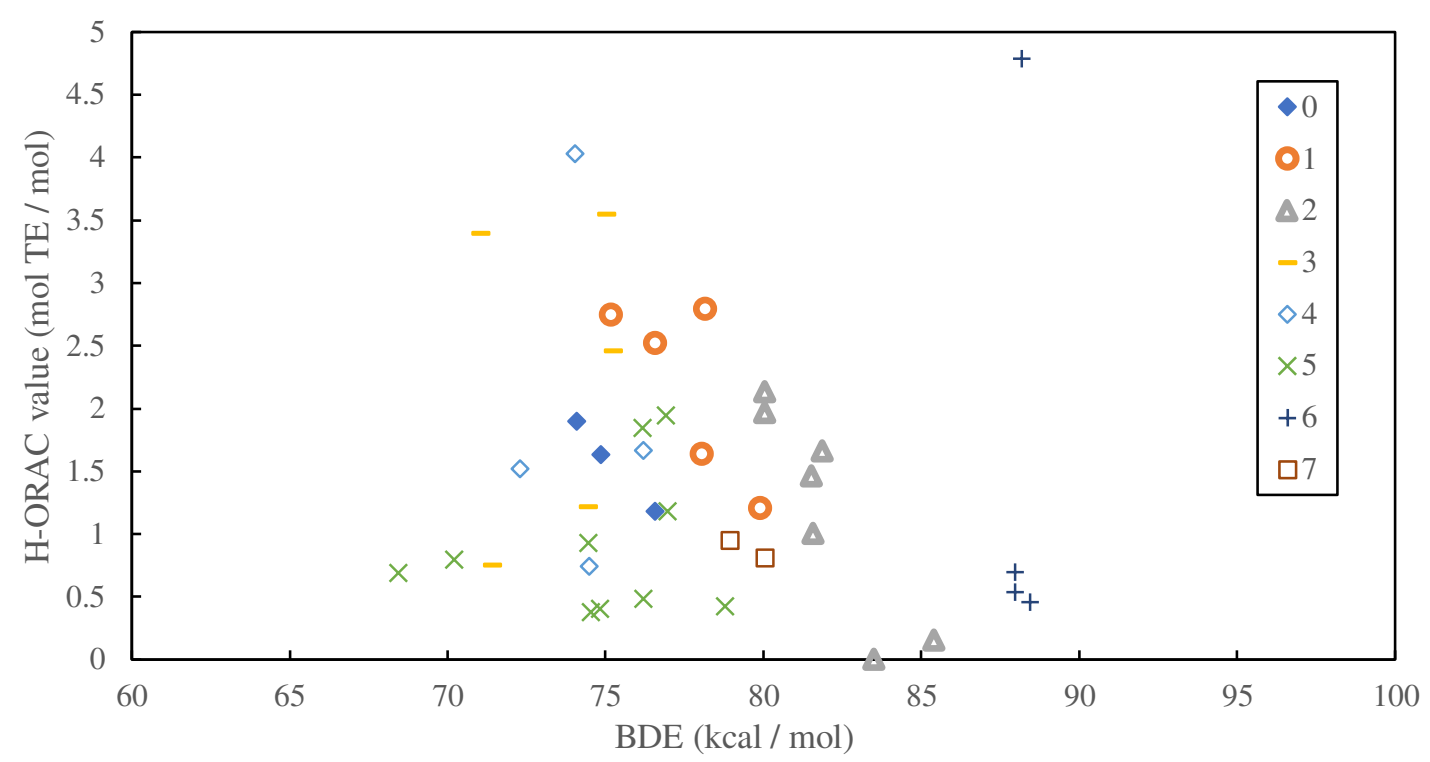

Fig. 1 Correlation between the minimum bond dissociation enthalpy (BDE) and hydrophilic oxygen radical absorbance capacity (H-ORAC) values

\subsection{Analysis}

We show the results of the stratified and clusterwise linear regression analysis in Fig. 2, Table 2. The statistical analyses were performed for groups 1 and 2 for which a linear relationship was observed. 


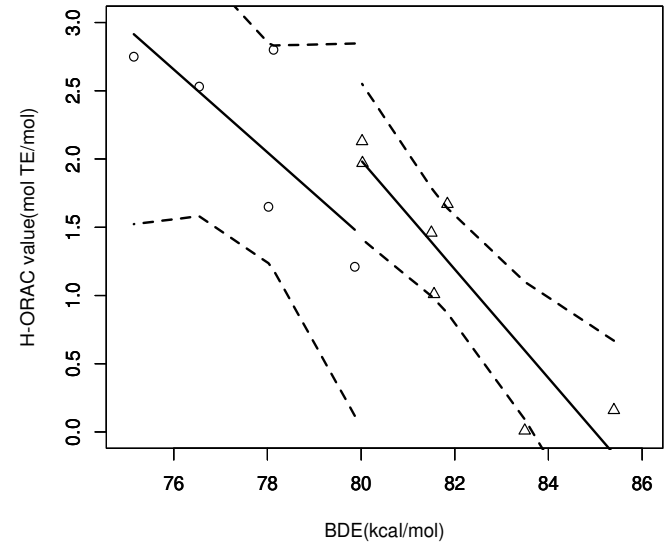

(a) Stratified linear regression analysis

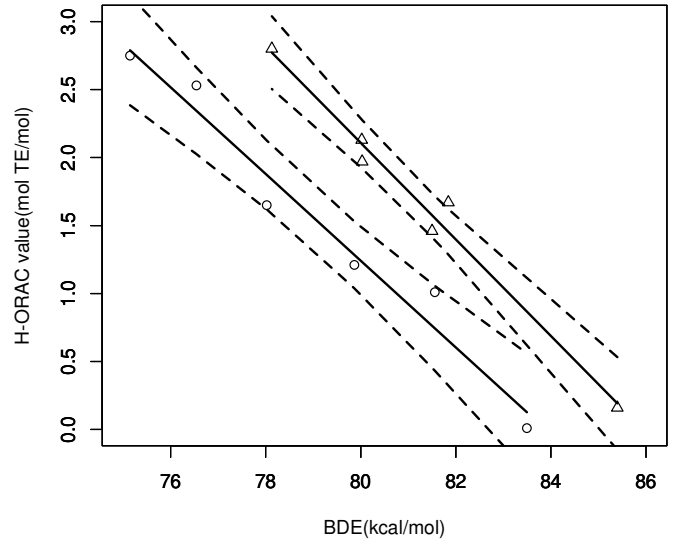

(b) Clusterwise linear regression analysis

Fig. 2 (a) Stratified linear regression analysis and (b) clusterwise linear regression analysis of the bond dissociation enthalpy (BDE) and hydrophilic oxygen radical absorbance capacity (H-ORAC). The solid lines are regression lines, while the dashed curves are 95\% confidence bands. In (a), $\bigcirc$ and $\triangle$ represent compounds that belong to neighborings 1 and 2, respectively. In (b), $\bigcirc$ and $\Delta$ represent compounds that belong to clusters 1 and 2, respectively.

Table 2 Regression Coefficients ${ }^{a}$

(a) Stratified linear regression analysis

\begin{tabular}{|c|c|c|c|c|c|c|c|c|}
\hline & \multicolumn{3}{|c|}{ Neighboring 1 } & \multicolumn{3}{c|}{ Neighboring 2 } \\
\hline Coefficients & Estimate & Std. error & t-value & p-value & Estimate & $\begin{array}{c}\text { Std. } \\
\text { error }\end{array}$ & $t$-value & $p$-value \\
\hline Intercept & $\begin{array}{c}25.73 \\
(-11.802,63.262)\end{array}$ & 11.79 & 2.18 & 0.12 & $\begin{array}{c}33.89 \\
(16.20,51.58)\end{array}$ & 6.88 & 4.93 & 0.004 \\
\hline BDE & $\begin{array}{c}-0.30 \\
(-0.788,0.180)\end{array}$ & 0.15 & -2.00 & 0.14 & $\begin{array}{c}-0.40 \\
(-0.61,-0.18)\end{array}$ & 0.08 & -4.75 & 0.005 \\
\hline
\end{tabular}

(b) Clusterwise linear regression analysis

\begin{tabular}{|c|c|c|c|c|c|c|c|c|}
\hline & \multicolumn{4}{|c|}{ Cluster 1 } & \multicolumn{5}{c|}{ Cluster 2 } \\
\hline Coefficients & Estimate & Std. error & t-value & $\mathrm{p}$-value & Estimate & Std. error & $\mathrm{t}$-value & $\mathrm{p}$-value \\
\hline Intercept & $\begin{array}{c}26.76 \\
(20.21,33.31)\end{array}$ & 2.36 & 11.34 & 0 & $\begin{array}{c}30.51 \\
(24.75,36.27)\end{array}$ & 2.07 & 14.71 & 0 \\
\hline BDE & $\begin{array}{c}-0.32 \\
(-0.40,-0.24)\end{array}$ & 0.03 & -10.70 & 0 & $\begin{array}{c}-0.36 \\
(-0.43,-0.28)\end{array}$ & 0.026 & -13.90 & 0 \\
\hline
\end{tabular}

${ }^{a}$ Estimate: least squared estimator and $95 \%$ confidence interval, std. error: standard error, $\mathrm{t}$-value: t-test statistics, BDE: bond dissociation enthalpy 


\section{Discussion}

Based on the clusterwise linear regression analysis, protocatechuic acid in group 1 and 3-(4hydroxyphenyl)propionic acid and 3-hydroxybenzoic acid in group 2 belong to different groups. Different labelling was performed to fill the voids in the data, and data on the H-ORAC values of group 1 compounds with a BDE of $80 \mathrm{kcal} / \mathrm{mol}$ or higher and group 2 compounds with a BDE of 82$84 \mathrm{kcal} / \mathrm{mol}$ were required. It is assumed that the $\mathrm{H}-\mathrm{ORAC}$ value is lower than that predicted from the $\mathrm{BDE}$ when there is an adjacent substituent because of steric hindrance. For some flavans, the H-ORAC value is independent of the minimum BDE. However, this is due to the difference between the reactivities of $\mathrm{CH}$ and $\mathrm{OH}$ and steric factors, although the $\mathrm{BDE}$ is the same. Moreover, because it is a substrate that reacts multiple times, it is assumed that H-ORAC value cannot be expressed by only the minimum BDE. A more detailed analysis is needed in this regard.

As shown in Tables 2(a) and 3(a), when stratified linear regression analysis is applied, the regression coefficient of BDE is significant for neighboring 2, but not for neighboring 1. In contrast, Tables 2(b) and 3(b) show that when clusterwise linear regression analysis is applied, the regression coefficient of $\mathrm{BDE}$ is highly significant for both clusters 1 and 2 . This implies that there is a strong linear relationship between the BDE and H-ORAC values in each cluster. In this study, because the true clusters, neighborings 1 and 2, are taken as the initial clusters, it would be desirable for the initial clusters and results of the analysis to be consistent with each other. In the future, such analysis will need to consider the accuracy of the structural optimisation method such as conformational search, accuracy of the BDE obtained at the PM7 level, and error in measurement. However, for phenols, it can be said that the tendency was confirmed by the BDE, which is greatly affected by the number of adjacent substituents of $\mathrm{OH}$ having the minimum BDE.

Comparison of Figs. 2(a) and 2(b) shows that clusters 1 and 2 include most compounds of neighborings 1 and 2, respectively. Thus, clusters 1 and 2 can reasonably characterize neighborings 1 and 2, respectively. However, a few compounds show different tendencies. Specifically, regarding the classification of phenols, the tendency of the category can be seen by classifying the substituent at the ortho position, although another factor is the effect of the substituent at the para or meta position. The $\mathrm{H}-\mathrm{ORAC}$ value is more than that predicted from the effect of the BDE of the phenolic hydroxyl group. Protocatechuic acid and 4-hydroxybenzoic acid also have a carbonyl group at the para position of ArOH that improves the H-ORAC value; hence, they deviate from the normal trend of group 2 and are situated above the regression line. It is also possible that the substance causing the misclassification has an extended regression line. This substance can be classified if a BDE threshold of around 80 $\mathrm{kcal} / \mathrm{mol}$ is taken into consideration.

\section{Conclusions}

By analysing the data for the main antioxidants found in wine, the trend in the H-ORAC values was 
determined using the calculated structures and BDEs of groups classified according to the presence or absence of oxygen functional groups in the ortho position of phenol. Clusterwise linear regression analysis showed that the regression coefficient of BDE is highly significant for both groups 0 (phenols di-substituted at the ortho position) and 1 (phenols mono-substituted at the ortho position), which implies that there is a strong linear relationship between the BDE and H-ORAC values in each homogenous cluster. Among the thermodynamic parameters calculated in this study, IP and PA did not show a clear trend like BDE. To analyse a wider range of compounds in the future, various indicators, such as steric factors and solubility, are needed. Flavans and indoles are important ingredients that are also found in food and physiologically active substances; hence, we will study them in more detail in the future.

\section{Conflicts of interest/Competing interests (include appropriate disclosures)}

There are no conflicts of interest to declare.

\section{Acknowledge}

We would like to thank Editage (www.editage.jp) for the English language editing.

\section{References}

1. Lee J, Koo N, Min DB (2004) Reactive oxygen species, aging, and antioxidative nutraceuticals Compr Rev Food Sci Food Saf 3:21-33. https://doi.org/10.1111/j.1541-4337.2004.tb00058.x.

2. Litwinienko G, Ingold KU (2007) Solvent effects on the rates and mechanisms of reaction of phenols with free radicals Acc Chem Res 40:222-230. https://doi.org/10.1021/ar0682029.

3. Galian RE, Litwinienko G, Pérez-Prieto J, Ingold KU (2007) Kinetic solvent effects on the reaction of an aromatic ketone $\pi, \pi^{*}$ triplet with phenol. Rate-retarding and rate-accelerating effects of hydrogenbond acceptor solvents J Am Chem Soc 129:9280-9281. https://doi.org/10.1021/ja071716y.

4. Litwinienko G, Ingold KU (2004) Abnormal solvent effects on hydrogen atom abstraction. 2. Resolution of the curcumin antioxidant controversy. The role of sequential proton loss electron transfer J Org Chem 69:5888-5896. https://doi.org/10.1021/j0049254j.

5. Antolovich M, Prenzler PD, Patsalides E, McDonald S, Robards K (2002) Methods for testing antioxidant activity Analyst 127:183-198. https://doi.org/10.1039/b009171p.

6. Huang D, Ou B, Prior RL (2005) The chemistry behind antioxidant capacity assays J Agric Food Chem 53:1841-1856. https://doi.org/10.1021/jf030723c.

7. Huang D, Ou B, Hampsch-Woodill M, Flanagan JA, Deemer EK (2002) Development and validation of oxygen radical absorbance capacity assay for lipophilic antioxidants using randomly methylated $\beta$ - 
cyclodextrin as the solubility enhancer J Agric Food Chem 50:1815-1821. https://doi.org/10.1021/jf0113732.

8. Cao G, Alessio HM, Cutler RG (1993) Oxygen-radical absorbance capacity assay for antioxidants Free Radical Biol Med 14:303-311. https://doi.org/10.1016/0891-5849(93)90027-R.

9. Watanabe J, Oki T, Takebayashi J, Yamasaki K, Takano-Ishikawa Y, Hino A, Yasui A (2012) Method validation by interlaboratory studies of improved hydrophilic oxygen radical absorbance capacity methods for the determination of antioxidant capacities of antioxidant solutions and food extracts Anal Sci 28:159. https://doi.org/10.2116/analsci.28.159.

10. Prior RL, Hoang H, Gu L, Wu X, Bacchiocca M, Howard L, Hampsch-Woodill, Huang D, Ou B, Jacob R (2003) Assays for hydrophilic and lipophilic antioxidant capacity (oxygen radical absorbance capacity $\left.\left(\mathrm{ORAC}_{\mathrm{FL}}\right)\right)$ of plasma and other biological and food samples J Agric Food Chem 51:32733279. https://doi.org/10.1021/jf0262256.

11. Prior RL, Wu X, Schaich K (2005) Standardized methods for the determination of antioxidant capacity and phenolics in foods and dietary supplements J Agric Food Chem 53:4290-4302. https://doi.org/10.1021/jf0502698.

12. Zhang D, Liu Y, Chu L, Wei Y, Wang D, Cai S, Zhou F, Ji B (2013) Relationship between the structures of flavonoids and oxygen radical absorbance capacity values: A quantum chemical analysis J Phys Chem A 117:1784-1794. https://doi.org/10.1021/jp307746c.

13. Villaño D, Fernández-Pachón MS, Troncoso AM, García-Parrilla MC (2005) Comparison of antioxidant activity of wine phenolic compounds and metabolites in vitro Anal Chim Acta 538:391398. https://doi.org/10.1016/j.aca.2005.02.016.

14. Rodriguez-Naranjo MI, Moyá ML, Cantos-Villar E, García-Parrilla MC (2012) Comparative evaluation of the antioxidant activity of melatonin and related indoles J Food Compos Anal 28:16-22. https://doi.org/10.1016/j.jfca.2012.07.001.

15. Singleton VL, Orthofer R, Lamuela-Raventós RM (1999) Analysis of total phenols and other oxidation substrates and antioxidants by means of folin-ciocalteu reagent Methods Enzymol 299:152-178. https://doi.org/10.1016/S0076-6879(99)99017-1.

16. Reiter RJ, Tan D-X, Mayo JC, Sainz RM, Leon J, Czarnocki Z (2003) Melatonin as an antioxidant: Biochemical mechanisms and pathophysiological implications in humans Acta Biochim Pol 50:11291146. https://doi.org/10.18388/abp.2003_3637.

17. Ordoudi SA, Tsimidou MZ, Vafiadis AP, Bakalbassis EG (2006) Structure-DPPH' scavenging activity relationships: Parallel study of catechol and guaiacol acid derivatives J Agric Food Chem 54:57635768. https://doi.org/10.1021/jf060132x.

18. Shen L, Zhang H-Y, Ji H-F (2008) A thermodynamic investigation of DPPH radical-scavenging mechanisms of folates $\mathrm{J}$ Mol Struct: THEOCHEM 856:119-123. https://doi.org/10.1016/j.theochem.2008.01.023. 
19. Ji H-F, Tang G-Y, Zhang H-Y (2005) Theoretical elucidation of DPPH radical-scavenging activity difference of antioxidant xanthones QSAR Comb Sci 24:826-830. https://doi.org/10.1002/qsar.200430917.

20. Slavova-Kazakova AK, Angelova, SE, Veprintsev TL, Denev, P, Fabbri D, Dettori MA, Kratchanova M, Naumov VV, Trofimov AV, Vasilév RF, Delogu G, Kancheva VD (2015) Antioxidant potential of curcumin-related compounds studied by chemiluminescence kinetics, chain-breaking efficiencies, scavenging activity (ORAC) and DFT calculations Beilstein J Org Chem 11:1398-1411. https://doi.org/10.3762/bjoc.11.151.

21. Žuvela P, David J, Yang X, Huang D, Wong MW (2019) Non-linear quantitative structure-activity relationships modelling, mechanistic study and in-silico design of flavonoids as potent antioxidants Int J Mol Sci 20:2328. https://doi.org/10.3390/ijms20092328.

22. Späth H (1992) Mathematical algorithms for linear regression. Academic Press, San Diego.

23. Späth H (1979) Algorithm 39 Clusterwise linear regression Computing 22:367-373. https://doi.org/10.1007/BF02265317.

24. Späth H (1982) A fast algorithm for clusterwise linear regression Computing 29:175-181. https://doi.org/10.1007/BF02249940.

25. Vicari D, Vichi M (2013) Multivariate linear regression for heterogeneous data J Appl Stat 40:12091230. https://doi.org/10.1080/02664763.2013.784896.

26. Vainio MJ, Johnson MS (2007) Generating conformer ensembles using a multiobjective genetic algorithm J Chem Inf Model 47:2462-2474. https://doi.org/10.1021/ci6005646.

27. Stewart JJP (1990) MOPAC: A semiempirical molecular orbital program J Comput-Aided Mol Des 4:1-103. https://doi.org/10.1007/BF00128336.

28. Bartolome B (2004) Extending Applicability of the Oxygen Radical Absorbance Capacity (ORACFluorescein) Assay J Agric Food Chem 52:48-54. https://doi.org/10.1021/jf0305231.

29. Naguib YM (2000) A fluorometric method for measurement of oxygen radical-scavenging activity of water-soluble antioxidants Anal Biochem 284:93-98 https://doi.org/10.1006/abio.2000.4691.

30. Řezáč J, Fanfrlík J, Salahub D, Hobza P (2009) Semiempirical quantum chemical PM6 method augmented by dispersion and H-bonding correction terms reliably describes various types of noncovalent complexes J Chem Theory Comput 5:1749-1760. https://doi.org/10.1021/ct9000922.

31. Grimme S (2004) Accurate description of van der Waals complexes by density functional theory including empirical corrections J Comput Chem 25:1463-1473. https://doi.org/10.1002/jcc.20078.

32. Bartmess JE (1994) Thermodynamics of the electron and the proton J Phys Chem 98:6420-6424. https://doi.org/10.1021/j100076a029.

33. Okawa M, Kinjo J, Nohara T, Ono M (2001) DPPH (1,1-diphenyl-2-picrylhydrazyl) radical scavenging activity of flavonoids obtained from some medicinal plants Biol Pharm Bull 24:1202-1205. https://doi.org/10.1248/bpb.24.1202. 
34. Kondo K, Kurihara M, Fukuhara K, Tanaka T, Suzuki T, Miyata N, Toyoda M (2000) Conversion of procyanidin B-type (catechin dimer) to A-type: Evidence for abstraction of C-2 hydrogen in catechin during radical oxidation Tetrahedron Lett 41:485-488. https://doi.org/10.1016/S0040-4039(99)020973. 


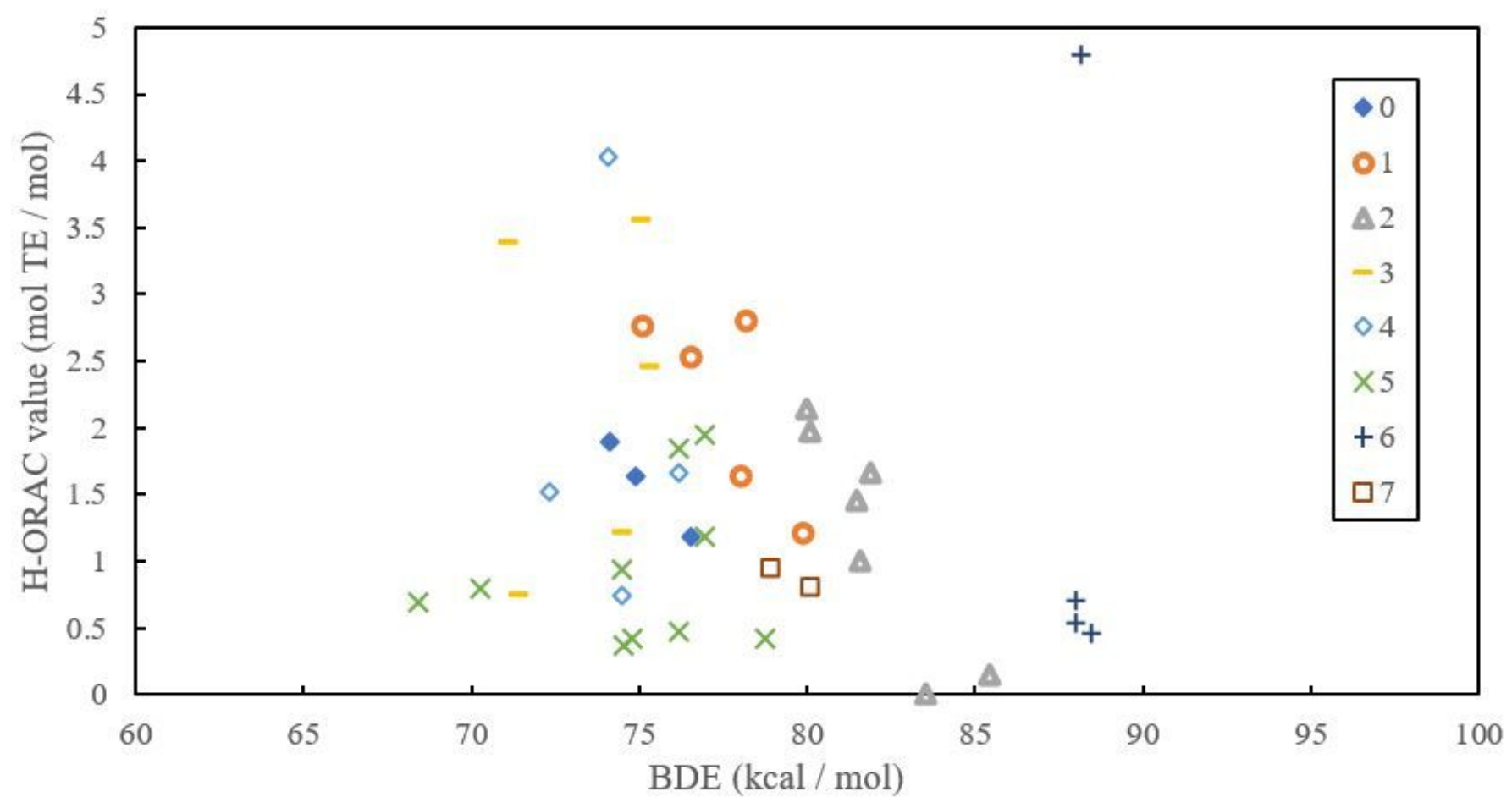

Figure 1

Correlation between the minimum bond dissociation enthalpy (BDE) and hydrophilic oxygen radical absorbance capacity (H-ORAC) values

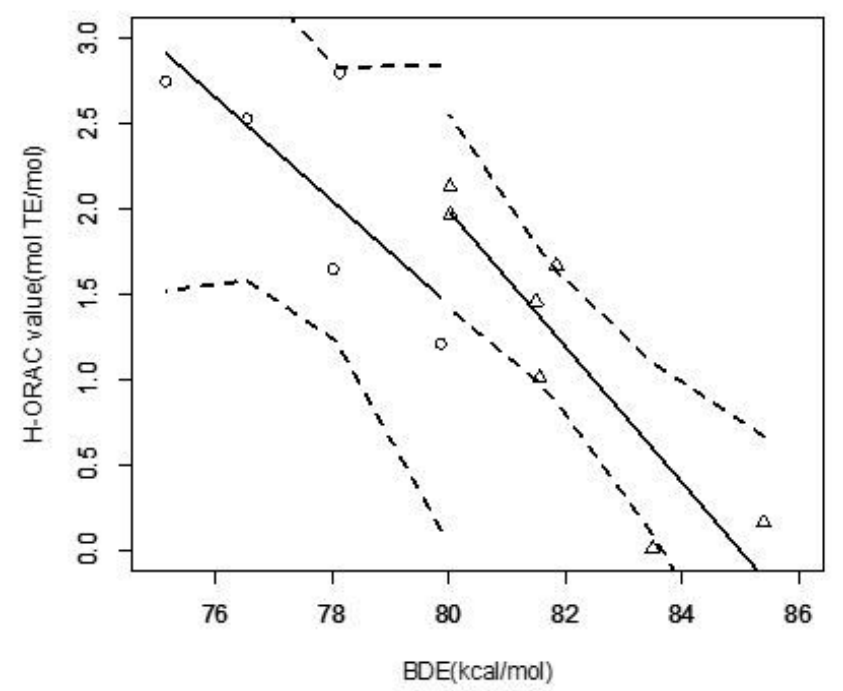

(a) Stratified linear regression analysis

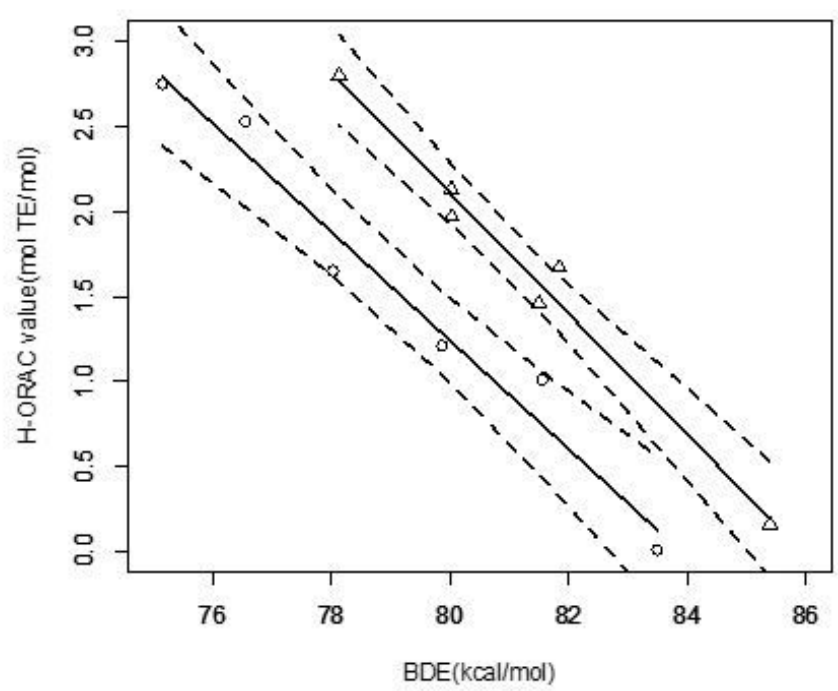

(b) Clusterwise linear regression analysis 
Figure 2

(a) Stratified linear regression analysis and (b) clusterwise linear regression analysis of the bond dissociation enthalpy (BDE) and hydrophilic oxygen radical absorbance capacity (H-ORAC). The solid

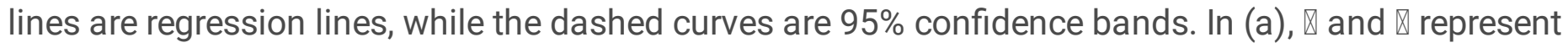
compounds that belong to neighborings 1 and 2 , respectively. In (b), $\nabla$ and $\nabla$ represent compounds that belong to clusters 1 and 2, respectively.

\section{Supplementary Files}

This is a list of supplementary files associated with this preprint. Click to download.

- SI.pdf 